\title{
RESTAURACIÓN EN EXPLOTACIONES DE MINAS CALIZA
}

\author{
Yeimy Consuelo Sarmiento Mora \\ Estudiante Maestría en Biología. Universidad Nacional. \\ Nidia Yaneth Torres Merchán \\ Estudiante Maestría en Docencia de la Química. Universidad Pedagógica Nacional. \\ yanethtorres3@hotmail.com
}

Manizales, 2008-08-26 (Rev. 2008-11-20)

\section{RESUMEN}

El trabajo que se presenta a continuación constituye un plan de restauración en terrenos que han sido utilizados para la explotación de caliza en la empresa Acerías Paz del Rio. Esta propuesta es producto de la previa caracterización de plantas superiores que realizaron las autoras en sitios donde se realizó la explotación de minas caliza (Chameza, Belencito y Mal Sitio). Este estudio tiene en cuenta tres componentes: el suelo, la vegetación y el trabajo con estudiantes.

\section{PALABRAS CLAVE}

Restauración, minas calizas, explotación minera, sucesiones vegetales.

\section{RESTORATION IN LIMESTONE MINES EXPLOITATION}

\begin{abstract}
The present work constitutes a restoration plan in areas that have been used for the exploitation of limestone in the Acerias Paz del Rio Company. This proposal is the product of a previous characterization of superior plants carried out by the authors in places where the exploitation of limestone mines took place (Chameza, Belencito and Mal Sitio). This study considers three components: soil, vegetation and the work with students.
\end{abstract}

\section{KEY WORDS}

Restoration, limestone mines, mining exploitation, vegetable successions.

\section{PRESENTACIÓN}

\section{DESCRIPCIÓN DEL SITIO DE ESTUDIO}

Los yacimientos de minas calizas que explota Acerías Paz del Rio, están situados en las propias instalaciones de Belencito, cerca de $2 \mathrm{~km}$, se trata de un yacimiento sedimentario marino del cretáceo inferior. (Amórtegui 1998). El sitio específico de 
estudio corresponde a terrenos aledaños a los yacimientos de minas calizas en Chameza, Belencito y Malsitio.

Dentro de los rasgos fisiográficos se destaca un relieve con forma abrupta con presencia de rocas de caliza y arenisca; según Heves (1985) la caliza encontrada corresponde a una biomicrita, una caliza químicamente pura constituida por fragmentos de fósiles calcáreos. Entre los minerales de residuo insoluble domina el cuarzo la arcilla y la glauconita.

Los suelos se caracterizan por ser superficies de alta pedregosidad, descubiertos con drenaje externo muy rápido, donde hay presencia de afloramientos rocosos, un contenido de materia orgánica bajo aproximadamente el 5\%, raicillas abundantes, y fertilidad baja. P. h básicos (Sarmiento \& Torres 2003).

La vegetación estudiada en los alrededores de las minas calizas de Acerías Paz del Rio, se puede asignar a una zona de vida andina que obedece a factores topográficos y a cambios climáticos asociados a los anteriores, la vegetación presenta formas achaparradas, unas estratificadas de tipo herbáceo asociada a pequeños grupos mono específicos.

\section{PROCESO DE RESTAURACIÓN EN LAS CANTERAS}

Para iniciar un programa de restauración es necesario:

- Comenzar los tratamientos en los puntos y franjas con mayor tasa de restauración formando focos y corredores de actividad biológica.

- Introducir las especies vegetales en el orden de sucesión y en la posición ambiental que les corresponde induciendo y acelerando la sucesión natural.

- Crear una red de sustentación a través del área a restaurar, en donde los focos y franjas de restauración engloben las áreas más alteradas a restaurar luego y aquellas donde se mantendrá la alteración (el uso).

La empresa Acerías Paz de Rio S.A ha puesto en práctica, desde el inicio de sus operaciones, métodos de explotación adecuados, buscando mantener y mejorar el estado de los terrenos aledaños mediante las labores de reforestación, recuperación morfológica y paisajística contemplados en el plan de mejoramiento ambiental diseñados para cada zona donde se encuentran las minas.

Para la fase de retrollenados se trabajan alturas de terrazas de 5 metros con el fin de garantizar su estabilidad y facilitar su 'revegetalización'; se disponen los estériles de tal manera que el perfil generado se ajuste a la morfología del terreno original; se construye un filtro en roca sobre el piso, sobre la cual se realiza el retrollenado para recoger las aguas filtradas, y un sistema general de cunetas para la recogida de las aguas externas de la zona y, también, para cada terraza.

El material estéril que se obtiene durante la explotación de la caliza está conformado principalmente por lutitas con delgadas intercalaciones calcáreas, los estériles -producto de la explotación- se disponen retro llenando las depresiones de la explotación a tajo abierto.

A partir de la flora caracterizada en las áreas de estudio, se formulan tratamientos de restauración en combinación con herramientas físicas y químicas con especies nativas que aceleran el proceso para garantizar tanto una cobertura significativa 
como la recuperación del hábitat en el mediano plazo, junto con la estabilización del sustrato.

En primer lugar, se debe realizar un diagnóstico rápido de la cantera identificando lo siguiente: áreas inestables, áreas estables, tipos de erosión (laminar, cárcavas, desplomes), focos de regeneración espontánea, drenajes, terracetas naturales.

- Se zonificarán estas áreas con los siguientes procedimientos: cordones físicos de contención, barreras vegetales de contención de escorrentía, focos de expansión de la vegetación, focos de fertilización estratégica, implementación de estructuras artificiales de colonización.

- Se iniciará el tratamiento con todas las obras físicas y se realizará seguimiento de las mismas para identificar sus resultados, ajustando y adecuando aquellas que no hayan funcionado según lo previsto.

- Terminados los ajustes se procederá a la fertilización.

- Se implementarán barreras de contención de escorrentía.

- Finalmente se implementarán los focos de expansión de la vegetación.

La distribución de cada uno de los anteriores tratamientos debe enfocarse en puntos en donde es más viable la restauración, micro topografía adecuada, humedad edáfica y estabilidad del sustrato.

La implementación de estas áreas dentro de la cantera permiten emprender una restauración que, una vez iniciada artificialmente, se retroalimenta positivamente y por sí sola en un término indefinido de tiempo, aumentando espontáneamente la tasa de modificación y la estabilidad del medio físico con cada nueva etapa y asegurando la marcha del terreno hacia su eventual recuperación.

Se consigue así frenar el avance de la erosión, controlar el aporte de sedimentos a la red hidrográfica, conservar cantidad y calidad del agua de las microcuencas afectadas y recuperar las canteras.

\section{PROGRAMA SUELO}

En un programa de restauración se intenta rehabilitar la zona afectada de forma que el suelo adquiera las propiedades mínimas para albergar vida animal y vegetal. Para llevar a cabo una restauración se debe seguir una serie de etapas que empiezan con evaluación de las características biofísicas del área, para luego determinar los usos potenciales de esta.

En la restauración de suelos se desarrollan métodos como el retrollenado de estériles, que son adecuados con la construcción de filtros, zanjas y canales de desvió, esta disposición se inicia por el pie avanzando hacia arriba, dejando talud y bermas de más de $5 \mathrm{~m}$ de ancho cada $10 \mathrm{~m}$ a $25 \mathrm{~m}$ de altura; cada talud se protege con vegetación para asegurar la resistencia a la erosión.

\section{ACTIVIDADES}

- Retrollenado de estériles

- Realización de taludes y bermas

- Elaboración de terrazas. 


\section{CONTROL DE EROSIÓN}

Un sistema que contribuye a contrarrestar la erosión severa ocasionada por la explotación minera a cielo abierto es el sistema Gradoh ${ }^{1}$, el cual se podría aplicar a las minas caliza de Belencito; este sistema, que se emplea a gran escala en Italia, Norte de África, India, Japón y otros países, para la restauración de terrenos muy erosionados, ha dado magníficos resultados. El método consiste en trazar curvas de nivel a una equidistancia vertical de 2 a $4 \mathrm{~m}$. Según la pendiente sobre las curvas de nivel, se abren zanjas o pequeñas terrazas de $0.60 \mathrm{~m}$ de ancho por $0.40 \mathrm{~m}$ de profundidad, 2 a $6 \mathrm{~m}$ de longitud y se deja un dique divisor de $40 \mathrm{~cm}$ entre zanja y zanja. La tierra extraída se coloca sobre el borde de la zanja del lado de la pendiente. Sobre este borde de tierra removida se plantan los árboles con el espaciamiento deseado.

Las razones técnicas del método son:

- Detener inmediatamente la erosión ocasionada por el escurrimiento del agua de lluvia.

- Colectar, retener e infiltrar el agua proveniente de las precipitaciones fluviales para proporcionar humedad a la vegetación en la época de sequía.

- Permitir con cierta altitud el desarrollo de los árboles, al quedar en un terreno removido.

- Proporcionar protección y vigilancia. Es preferible emplear el presupuesto en proteger la repoblación natural o las plantaciones naturales que extender la reforestación sin protegerla.

Algunas especies recomendadas para el control de la erosión en el sitio de estudio son las siguientes:

- Baccharis latifolia: excelente para la recuperación de suelos desnudos, control de taludes y surcos.

- Cortaderia nítida: ideal para la construcción de pequeños microclimas y la protección y formación de pequeños enclaves de suelo en regeneración.

- Chusquea scandens: fundamentalmente en la protección de márgenes, cañadas y estabilización de taludes.

- Dodonea viscosa: restauración de focos de erosión severa y afloramiento rocoso, restauración en zonas secas.

- Solanum lycioides: restauración de focos de erosión severa protección de taludes.

- Macleania rupestris: Como herramienta agroforestal, como barrera contra vientos y contra heladas.

\section{Especies dinamogenéticas:}

Luego de lograr la adecuación del microclima y el sustrato con herramientas físicas y químicas que simulan el efecto sucesional de la vegetación, se aseguran condiciones propicias para el establecimiento de las especies dinamogenéticas como las siguientes: 


\begin{tabular}{|c|c|c|}
\hline ESPECIE & PAPEL ECOLÓGICO & DISPERSIÓN \\
\hline Piper bogotensis & $\begin{array}{c}\text { Conservación, avifauna, } \\
\text { medicinal }\end{array}$ & Estacas \\
\hline Prunus serotina & Medicinal & Semillas \\
\hline Rubus floribundus & Cerca viva, avifauna & Semillas, estacas \\
\hline Solanum lycioides & Ornamental, control de erosión & Semillas \\
\hline $\begin{array}{c}\text { Xylosma } \\
\text { spiculiferum }\end{array}$ & Cerca viva, control de erosión & Semillas \\
\hline Alnus acuminata & Fijador de nitrógeno, madera & Semillas \\
\hline Baccharis latifolia & $\begin{array}{c}\text { Restauración de suelos, } \\
\text { medicinal }\end{array}$ & Semillas \\
\hline Macleania rupestris & $\begin{array}{c}\text { Conservación, cerca viva, } \\
\text { avifauna }\end{array}$ & Semillas \\
\hline $\begin{array}{c}\text { Chusquea } \\
\text { scandens }\end{array}$ & \begin{tabular}{c} 
Cerca viva \\
\hline Dodonea viscosa
\end{tabular} & Control de erosión, cerca viva \\
\hline Miconia & Conservación & Semillas \\
\hline squamulosa & Medicinal, cerca viva & Semillas \\
\hline Agave americana & Semillas \\
\hline Opuntia shumanii. & Cerca viva, control de erosión & Semilla, tallo \\
\hline
\end{tabular}

Tabla 1. Especies dinamogenéticas que contribuyen en la adecuación del microclima.

\section{PROGRAMA RE VEGETACIÓN}

La restauración implica un diseño exacto del sistema de plantación que incluye realizar la preparación del terreno y la plantación cuidadosa de las plantas nativas, buscando que las especies se adapten al sitio para obtener un buen crecimiento y alta calidad.

En el caso de Acerías Paz del Rio se sugiere revegetalizar con las especies autóctonas que se desarrollaban normalmente en la zona, procurar acondicionar el terreno a su perfil primitivo para que cualquier alteración orográfica no ocasione nuevos problemas de restauración, tales como torrenteras, erosión, etc. (Salamanca. 1993).

La revegetalizacion se realiza siguiendo el patrón básico de la sucesión vegetal "patrón seral básico" la técnica a aplicar es la de plantación seral, la cual consiste en introducir en cada sitio las especies y morfotipo de la etapa sucesional inmediatamente posterior al estadio sucesional actual. Se efectúan varias plantaciones sucesivas, distanciada por lapso de años cada una introduciendo los juveniles de una etapa más avanzada.

Teniendo en cuenta los análisis de suelo del sitio, se encontró deficiencia de nutrientes básicos, como por ejemplo la materia orgánica que le proporciona nitrógeno a las plantas; por tal razón, se propone realizar la restauración con plantas que capturan nitrógeno y, de esta manera, incrementar la cantidad de este elemento en el suelo. Estas plantas pueden ser gramíneas (como el Halcus lanatus y Cortaderia nítida) que tienen crecimiento rápido y son tolerables a suelos pobres; igualmente tienen gran capacidad de invasión, logrando así la estabilidad del suelo. Luego se puede proceder a la siembra de especies arbustivas como Hayuelo, jarilla, 
Ciro, etc. Una vez la sucesión anterior manifieste su desarrollo y, finalmente, la siembra de árboles como el Mangle, Dividivi, Cucharo.

Las plantas más útiles son aquellas que presentan altas tasas de renovación (que aporta a la formación del suelo) y reproducción rápida y profusa (que les permite mantenerse a través de las perturbaciones devastadoras crónicas de las etapas iniciales). Estas especies colonizan los sitios más propicios y, a su alrededor, otras pueden establecerse.

\section{ACTIVIDADES}

- Formación de materia orgánica en el suelo

- Siembra de herbáceas

- Siembra de arbustos

- Siembra de árboles

- Control y seguimiento.

\section{ESPECIES VEGETALES SUGERIDAS PARA CADA ÁREA}

Establecidos los cordones de contención físicos y las estructuras artificiales de colonización, se pueden plantar las siguientes especies:

- Cortaderia nítida

- Puya sp.

- Lycium sp (en lugares atmosféricamente secos)

- Dodonea viscosa (en lugares atmosféricamente secos)

- Baccharis latifolia

- Agave americana

Para barreras vegetales de contención de escorrentía: el establecimiento de especies con hábito de crecimiento reptante o rastrero, y formadoras de suelo aptas para afianzar los primeros milímetros de suelo, claves en el control de erosión.

- Pyracantha coccinea

- Myrica parvifolia

- Trifolium repens

- Rubus bogotensis

- Macleania rupestris

- Befaria resinosa

- Piper bogotensis

Para los focos de expansión de la vegetación se recomienda la utilización de especies con hábito de crecimiento del tipo trepador, caracterizadas por su rápido crecimiento y su capacidad de superar pendientes fuertes.

- Rubus sp

- Bidens pilosa

En focos de fertilización estratégica: la utilización de especies con oferta de frutos atractiva para las aves, con lo que se multiplica la lluvia de propágulos sobre el área tratada, al tiempo con la fertilización adicional procedente de las deyecciones.

- Rubus sp.

- Macleania rupestris 
- Xylosma speculiferum

- Hesperomeles $s p$

Establecer especies que formen una cobertura a nivel del suelo y creen un microclima más amable y así favorezca la proliferación de especies de etapas más avanzadas de la sucesión.

- Macleania rupestris

- Myrica parvifolia

El establecimiento de especies de tipo arbustivo sobre las etapas previas, que determinen el avance a una etapa posterior de la sucesión (dinamogenéticas).

- Baccharis sp

- Schinus molle

- Stevia lucida

- Baccharis bogotensis

- Solanum sp

Por último, se implementaran herramientas complementarias en los lugares más difíciles. A continuación se describen los medios físicos fáciles de implementar que pueden ser:

- Tinglados: pueden ser de alambre y guadua, sobre pendientes fuertes con planes de tierra sosteniendo vegetación rastrera y trepadora.

- Terracetas artificiales: de las que pueden cavarse y apisonarse con la sola ayuda de un azadón. El perímetro externo de cada terraceta debe ser lo más semicircular posible y afianzarse con material vegetal.

- Drenajes artificiales: en focos de erosión severa deben efectuarse obras de conducción consistentes en canales que conduzcan la escorrentía desviándola de los frentes de erosión y utilizándola para la irrigación de los puntos menos frágiles (siempre que sea posible).

- Descoles: zanjas de drenaje que recogen la escorrentía de exceso de las demás obras de infiltración y control de erosión, canalizándola hacia fuera del área tratada. La falta de descoles es uno de los peores y más frecuentes errores en el control de erosión.

- Trinchos: en las cárcavas pueden construirse trinchos con postes de ocal con una barrera delantera de precursores leñosos de los recomendados para suelos pesados (húmedos).

- Geotextiles: textiles biodegradables (fique preferiblemente) cubriendo a manera de tapete los lugares más afectados por escorrentía superficial. (Foster 1967).

\section{COMPLEMENTAR CON MEDIOS QUÍMICOS Y ORGÁNICOS}

- Sopas biológicas: soluciones químicas, microbiológicas, multisemilladas o mixtas, solas o en combinación con mulch (residuos vegetales picados), para fertilización e inducción sucesional de suelos degradados.

- Nódulos de difusión lenta: para equilibrar la deficiencia general de fósforo y otros elementos limitantes en terrenos desnudos y con pendientes fuertes (factores que dificultan la retención de nutrientes). Dependiendo del interés y valor de la recuperación de un sitio dado, puede aplicarse un sistema de fertilización consistente en la introducción de nódulos de gel embebido en soluciones concentradas, en agujeros dentro del sustrato a inducir (la difusión 
lenta del nódulo al sustrato evita el lavado rápido del fertilizante). Combinados con puntos de colonización vegetal, ayudan a conformar núcleos de restauración en micrositios difíciles (taludes, escarpes, grietas).

- Mezcla de material rocoso fragmentado con suelo o desechos orgánicos para estimular la formación de suelo y la colonización de vegetación: se aplica para los residuos de material de la cantera en las áreas planas y estables. Se puede introducir, en una segunda etapa, la implementación de lombricultura. (Salamanca \& Camargo 1993).

\section{PROCESO DE REGENERACIÓN}

El proceso de la regeneración es lento y demora en alcanzar tazas mayores en especial para las plántulas en donde las condiciones son hostiles. Las fluctuaciones de temperatura afectan drásticamente el balance hídrico de estos organismos de escaso volumen; poco a poco, en distintos puntos y granjas mas favorables, la vegetación y el suelo logra algún acumulado sobre el cual multiplicar sus ganancias; lentamente comienzan a construirse circuitos de regulación biótica regenerada gana control creciente sobre la temperatura humedad, concentraciones químicas estabilidad mecánica, etc.

Los sustratos expuestos son más favorables a la regeneración en la medida en que presentan:

- Riqueza mineral

- Proporción de materiales finos

- Humedad constante

- Menor pendiente

- Profundidad efectiva

- Mayor calor específico

- Menores fluctuaciones micro-climáticas (recalentamiento, desecación congelamiento)

Una fuente importante de nutrientes y partículas finas está en la escorrentía procedente de los suelos y la vegetación en laderas por encima de la cantera. La regeneración espontánea de la cantera es lenta, pues depende de los aportes que la vegetación pueda hacer a la formación del suelo. Sin embargo, las plantas acumulan poca materia orgánica, pues su productividad y crecimiento son lentos en las severas condiciones micro-climáticas de la cantera; por ello- se impone una estrategia de mejoramiento especial que posibilita el establecimiento de la vegetación, que abarca el cubrimiento -pasando por la adición de distintos materiales y sustancias (incluso suelo ya formado y procedente de otros sitios)hasta la adición de materiales vivos en forma de cultivos diversos de hongos, bacterias, lombrices, con lo cual se añade sustrato con actividad biológica ya incorporada.

Posteriormente de lograr cobertura vegetal lo siguiente es atraer a los dispersores de semillas (aves, insectos) en el establecimiento de especies con frutos y flores.

\section{TRABAJO CON ESTUDIANTES}

De acuerdo con lo expresado por Bermúdez (1995), con el aumento de la población y la disminución de los recurso naturales, es importante involucrar a los estudiantes en el desarrollo de proyectos de conservación, que incluya tanto el conocimiento de 
la flora y fauna existente en una región, como el desarrollo de estrategias de desarrollo sostenible que garanticen la sostenibilidad del entorno.

En esta parte se involucró a los estudiantes de grado Noveno del Colegio Técnico de Nazaret del municipio de Nobsa y se les orientó sobre técnicas de recolección de material vegetal, realización de parcelas y descripción de cada uno de los sitios de estudio. Esto permitió hacer un intercambio de aprendizajes, nombres científicos de las plantas, utilidades de las mismas, técnicas de muestreo, registro de datos, ubicación exacta de los sitios de muestreo, narración de algunas leyendas por parte de los alumnos y visualización del impacto ambiental que trae la explotación de minas caliza en su región. Esta experiencia permitió establecer un diálogo racional entre su propia perspectiva y la de sus compañeros respecto a la importancia del medio ambiente.

\section{ACTIVIDADES}

- Charlas de capacitación sobre impacto ambiental y explotación de minas caliza.

- Jornada de revegetalizacion.

- Monitoreo de especies vegetales sembradas: cada estudiante hizo revisión documental de la especie, donde aprende el nombre científico, propagación por semillas: esta información es compartida con los compañeros a través de cartelera. La finalidad de esta actividad es lograr que los estudiantes sientan la necesidad de un ambiente más natural y se preocupen por desarrollar acciones preventivas que permitan amortiguar el impacto que el hombre ha generado en la naturaleza

\section{A MANERA DE CONCLUSIÓN}

- En la actualidad, es de vital importancia enseñar a los jóvenes el significado de los recursos naturales para asegurar la conservación de los mismos, lo ideal es involucrar a los estudiantes en proyectos relacionados con la educación ambiental.

- En cualquier propuesta de restauración se debe tener en cuenta la vegetación natural que evidencia la compatibilidad entre los factores bióticos y abióticos.

- Los planes de restauración deben enfocase hacia una integración paisajística, la cual hace relación a la armonía que debe guardar la explotación minera con su entrono natural, teniendo en cuenta el apantallamiento natural, la revegetalización y empedradización.

- Se debe controlar la disposición de estériles y capa vegetal para evitar procesos erosivos, movimiento en masa y pérdida de capacidad reproductiva del suelo mediante un sistema de retrollenado dentro de las zonas explotadas, llevando actividades como el retirado y acopio de la tierra vegetal de las zonas explotadas y disponerlos de tal forma que permitan continuar con los proceso de reconstrucción del suelo.

\section{BIBLIOGRAFÍA}

- AMORTEGUI, J. 1998. Efecto ambiental de la mina caliza Belencito. Ingeniería y Geotecnia LTDA.

- GUTIÉRREZ, A. 1981. Etapas de reforestación conservacionismo y desarrollo del recurso forestal. México. 
- FOSTER, A. 1967. Métodos apropiados en conservación de suelos. .México.

- HEVES I. 1985. Minas caliza de Belencito.

- SARMIENTO \& TORRES. 2004. Caracterización preliminar de pantas superiores en terrenos aledaños aminas caliza. Tesis de grado Universidad Pedagógica y Tecnológica de Colombia. Tunja.

- SALAMANCA, B. 1993. Revitalización estratégica en la conservación del bosque alto andino. Tesis de grado. Universidad Javeriana. Santa fe de Bogotá.

\section{NOTAS}

1. GUTIERREZ, Alfonso. Etapas de restauración. México 1981. 